\title{
New Iridium Complex Coordinated with Tetrathiafulvalene Substituted Triazole-pyridine Ligand: Synthesis, Photophysical and Electrochemical Properties
}

\author{
Zhi-Gang Niu, Hui Xie, Li-Rong He, Kai-Xiu Li, Qing Xia, \\ Dong-Min Wu, Gao-Nan Li* \\ College of Chemistry and Chemical Engineering, Hainan Normal University, Haikou 571158, PR China \\ * Corresponding author: E-mail: ligaonan2008@163.com, \\ niuzhigang1982@126.com
}

Received: 13-01-2016

\begin{abstract}
A new iridium(III) complex based on the triazole-pyridine ligand with tetrathiafulvalene unit, $\left[\operatorname{Ir}(\mathrm{ppy})_{2}(\mathbf{L})\right] \mathrm{PF}_{6}(\mathbf{1})$, has been synthesized and structurally characterized. The absorption spectra, luminescent spectra and electrochemical behaviors of $\mathbf{L}$ and $\mathbf{1}$ have been investigated. Complex $\mathbf{1}$ is found to be emissive at room temperature with maxima at 481 and $510 \mathrm{~nm}$. The broad and structured emission bands are suggested a mixing of ${ }^{3} \mathrm{LC}\left({ }^{3} \pi-\pi^{*}\right)$ and ${ }^{3} \mathrm{CT}\left({ }^{3} \mathrm{MLCT}\right)$ excited states. The influence of iridium ion coordination on the redox properties of the TTF has also been investigated by cyclic voltammetry.
\end{abstract}

Keywords: Iridium(III) complexes; Tetrathiafulvalene; Triazole-pyridine ligands; Photoluminescence; Cyclic voltammetry

\section{Introduction}

For several decades, tetrathiafulvalene (TTF) and its derivatives were extensively developed by scientists in photofunctional materials ${ }^{1-8}$ because of their strongly electron-donating and attractive reversible redox properties. As a consequence, a large synthetic effort has also been devoted to the preparation of materials that exhibit synergy or coexistence between conductivity and luminescence. Coordination of TTF-containing ligands to transition metal centers is typically achieved by functionalizing TTF with nitrogen atom. ${ }^{9}$

Very recently, we have reported a new nitrogen-containing TTF-based ligand, 2-(1-(2-((4',5'-bis(methylthio)[2,2'-bi(1,3-dithiolylidene)]-4-yl)thio)ethyl)- $1 \mathrm{H}-1,2,3$ triazol-4-yl)pyridine ( $\mathbf{L}$ ). Ligand $\mathbf{L}$ was used as the polyp-

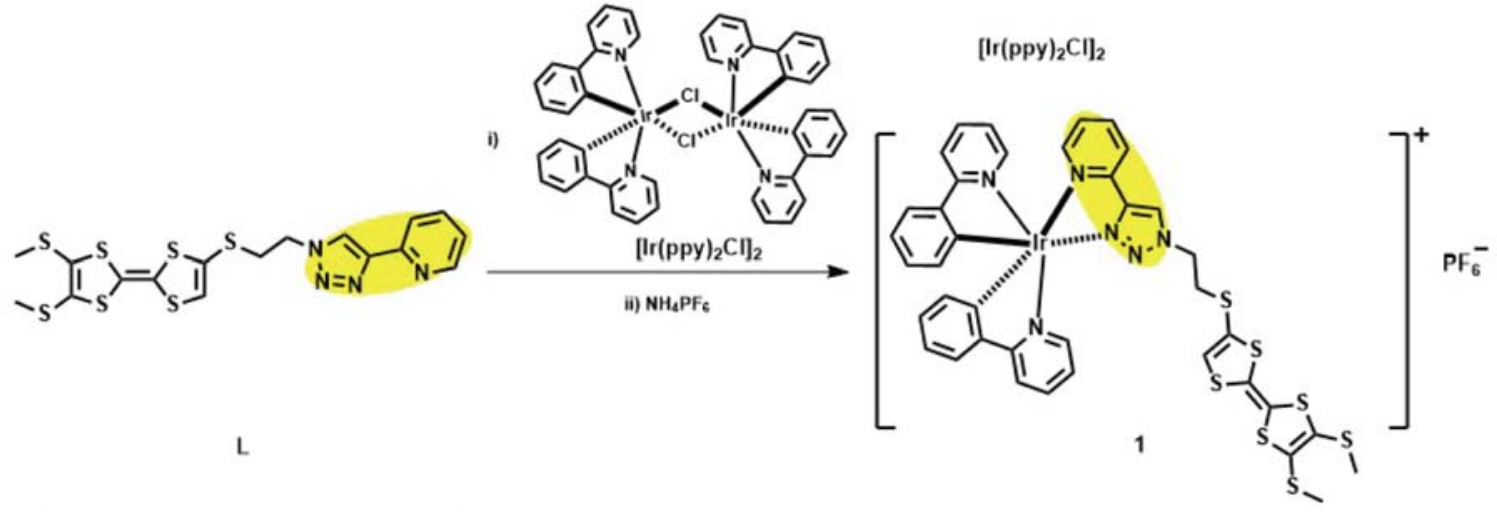

Scheme 1. Synthetic routes of $\operatorname{Ir}(\mathrm{III})$ complex 1. 
yridine $\mathrm{N}^{\wedge} \mathrm{N}$ ligand and binap/xantphos as diphosphines $\mathrm{P}^{\wedge} \mathrm{P}$ ligand to form two $\mathrm{Cu}(\mathrm{I})$ complexes, $[\mathrm{Cu}(\mathrm{I})$ (Binap) $(\mathrm{L})] \mathrm{BF}_{4}$ and $[\mathrm{Cu}(\mathrm{I})(\mathrm{Xantphos})(\mathrm{L})] \mathrm{BF}_{4}$, which exhibited advantageous electrochemical and photophysical properties. ${ }^{10}$ The results hence led us to further design other metal complexes based on the TTF-containing thioethylbridged triazole-pyridine ligand.

Iridium(III) complexes have widely been employed in organic light-emitting devices (OLEDs), as they have high phosphorescence quantum efficiency, long excitedstate lifetime and excellent color tenability. ${ }^{11-14}$ Therefore, the association of the redox-active TTF unit with cyclometalated iridium(III) complex is intriguing in coordination chemistry and material chemistry. In this work, we report the synthesis of a new bis-cyclometallated TTF-based iridium(III) complex with ppy as $\mathrm{C}^{\wedge} \mathrm{N}$ ligand, $\left[\operatorname{Ir}(\text { ppy })_{2}(\mathbf{L})\right] \mathrm{PF}_{6}(\mathbf{1})($ Scheme 1). Their electrochemical and photophysical properties are also investigated.

\section{Experimental}

\section{1. Materials and Measurements}

2-(1-(2-((4',5'-bis(methylthio)-[2,2'-bi(1,3-dithiolylidene)]-4-yl)thio)ethyl)-1H-1,2,3-triazol-4-yl)pyridine (L) was synthesized in our previous work, ${ }^{8}$ and an improved preparation method was used to synthesize the cyclometalated iridium chlorobridged dimer $\left[\operatorname{Ir}(\mathrm{ppy})_{2} \mathrm{Cl}\right]_{2}$ in good yield. ${ }^{15}$ All solvents were dried using standard procedures. Solvents used for electrochemistry and spectroscopy were spectroscopic grade.

${ }^{1} \mathrm{H}$ NMR and ${ }^{13} \mathrm{C}$ NMR spectra were recorded on a Bruker AM $400 \mathrm{MHz}$ instrument. Chemical shifts were reported in ppm relative to $\mathrm{Me}_{4} \mathrm{Si}$ as internal standard. FT-IR spectra were taken on a Nicolet 6700 FTIR spectrometer (400-4000 $\mathrm{cm}^{-1}$ ) with $\mathrm{KBr}$ pellets. ESI-MS spectra were recorded on an Esquire HCT-Agilent 1200 LC/MS spectrometer. The elemental analyses were performed on a Vario EL Cube Analyzer system. UV-vis spectra were recorded on a Hitachi U3900/3900H spectrophotometer. Fluorescence spectra were carried out on a Hitachi F-7000 spectrophotometer.

\section{2. Synthesis of $\left[\operatorname{Ir}(p p y)_{2}(L)\right] P_{6}(1)$}

A mixture of a dimer $\left[\operatorname{Ir}(\text { ppy })_{2} \mathrm{Cl}\right]_{2}(50 \mathrm{mg}, 46.5$ $\mu \mathrm{mol})$ and $\mathbf{L}(58 \mathrm{mg}, 93.0 \mu \mathrm{mol})$ was dissolved in $6 \mathrm{~mL}$ of $\mathrm{DCM}$ and $\mathrm{MeOH}(\mathrm{v} / \mathrm{v}=1: 1)$ and refluxed for $6 \mathrm{~h}$ under nitrogen. The orange-red solution was then cooled to room temperature and $\mathrm{NH}_{4} \mathrm{PF}_{6}(38 \mathrm{mg}, 0.23 \mathrm{mmol})$ was added to the solution. The mixture was stirred at room temperature for $4 \mathrm{~h}$, and then evaporated to dryness. The solid was purified by column chromatography with $\mathrm{DCM} / \mathrm{MeOH}(100$ : 1) eluent to afford pure product 1 (54 mg, Yield: $50.5 \%$ ) as a yellow solid. ${ }^{1} \mathrm{H}$ NMR $\left(400 \mathrm{MHz}, \mathrm{CDCl}_{3}\right): \delta 9.04(\mathrm{~s}$, $1 \mathrm{H}), 8.27(\mathrm{~d}, J=8.0 \mathrm{~Hz}, 1 \mathrm{H}), 7.99(\mathrm{t}, J=7.6 \mathrm{~Hz}, 1 \mathrm{H})$,
$7.90 \sim 7.92(\mathrm{~m}, 2 \mathrm{H}), 7.82(\mathrm{~d}, J=4.2 \mathrm{~Hz}, 1 \mathrm{H}), 7.65 \sim 7.79(\mathrm{~m}$, $6 \mathrm{H}), 7.53(\mathrm{~d}, J=5.6 \mathrm{~Hz}, 1 \mathrm{H}), 6.88 \sim 7.08(\mathrm{~m}, 6 \mathrm{H}), 6.40(\mathrm{~d}$, $J=7.2 \mathrm{~Hz}, 1 \mathrm{H}), 6.31(\mathrm{~d}, J=6.8 \mathrm{~Hz}, 1 \mathrm{H}), 5.97(\mathrm{~s}, 1 \mathrm{H}), 4.63$ $(\mathrm{t}, J=6.0 \mathrm{~Hz}, 2 \mathrm{H}), 3.12 \sim 3.15(\mathrm{~m}, 2 \mathrm{H}), 2.42(\mathrm{~s}, 6 \mathrm{H}) .{ }^{13} \mathrm{C}$ NMR $\left(100 \mathrm{MHz}, \mathrm{CDCl}_{3}\right): \delta 168.2,167.6,150.0,149.9$, $149.7,149.5,148.7,148.5,146.2,143.8,143.7,139.7$, $138.1,138.0,132.0,131.9,130.7,130.2,129.1,127.9$, 127.1, 126.6, 126.3, 124.8, 124.5, 123.5, 123.1, 122.8, 122.6, 121.6, 119.5, 119.4, 114.5, 108.9, 49.6, 34.8, 29.7; ESI-MS (m/z): $1001.0\left[\mathrm{M}-\mathrm{PF}_{6}^{-}\right]^{+}$. IR $\left(\mathrm{cm}^{-1}\right): \mathrm{v}=3442$ (m), $2922(\mathrm{w}), 2853(\mathrm{w}), 1608(\mathrm{~m}), 1475(\mathrm{~m}), 1422(\mathrm{~m})$, $1265(\mathrm{w}), 1100(\mathrm{w}), 842$ (s), $756(\mathrm{~m}), 556(\mathrm{w})$. Anal. calcd. For $\mathrm{C}_{39} \mathrm{H}_{32} \mathrm{~F}_{6} \mathrm{IrN}_{6} \mathrm{PS}_{7}$ : C 40.86, H 2.81, $\mathrm{N} 7.33$; found: $\mathrm{C}$ 40.95, H 2.96, N 7.45.

\section{3. Cyclic Voltammetry}

Cyclic voltammetry (CV) was performed on a $\mathrm{CHI}$ 1210B electrochemical workstation, with a glassy carbon electrode as the working electrode, a platinum wire as the counter electrode, an aqueous saturated calomel electrode (SCE) as the reference electrode, and $0.1 \mathrm{M} n-\mathrm{Bu}_{4} \mathrm{NClO}_{4}$ as the supporting electrolyte.

\section{Results and Discussion}

\section{1. Photophysical Properties}

\section{1. 1. Absorption Properties}

The absorption spectra of $\mathbf{L}$ and $\mathbf{1}$ in dichloromethane solution at room temperature are depicted in Fig. 1. For ligand $\mathbf{L}$ and complex $\mathbf{1}$, these strong absorption bands at a high energy $(\lambda<350 \mathrm{~nm})$ are assigned to spin-allowed intraligand $\left(\pi \rightarrow \pi^{*}\right)$ transitions of TTF-TzPy ligand (L) or ancillary ligand (ppy). The moderate absorption bands at lower energy (350-450 nm) correspond to intramolecular charge-transfer transition (ICT) for $\mathbf{L}^{16}$ and metal-to-ligand charge-transfer (MLCT, $\left.\mathrm{d} \pi(\mathrm{Ir}) \rightarrow \pi^{*}(\mathrm{~L})\right)$ transition for $\mathbf{1}$, respectively. ${ }^{17,18}$

\section{1. 2. Emission Properties}

The relative emission spectra of ligand $\mathbf{L}$ and complex 1 in degassed $\mathrm{CH}_{2} \mathrm{Cl}_{2}$ solution at room temperature are also given in Fig. 1. Upon excitation at $438 \mathrm{~nm}$, complex 1 displays two intense emission maxima at ca. 481 and $510 \mathrm{~nm}$. As for $\mathbf{L}$, the emission band occurs at about $462 \mathrm{~nm}\left(\lambda_{\mathrm{ex}}=363 \mathrm{~nm}\right)$. Therefore the vibronically structured emission of $\mathbf{1}$ is probably derived from a mixing of ${ }^{3} \mathrm{LC}\left({ }^{3} \pi-\pi^{*}\right)$ and ${ }^{3} \mathrm{CT}\left({ }^{3} \mathrm{MLCT}\right)$ excited states. ${ }^{19,20}$

\section{2. Electrochemical Properties}

The electrochemical behaviors of the ligand $\mathbf{L}$ and iridium complex 1 were investigated by cyclic voltamme- 


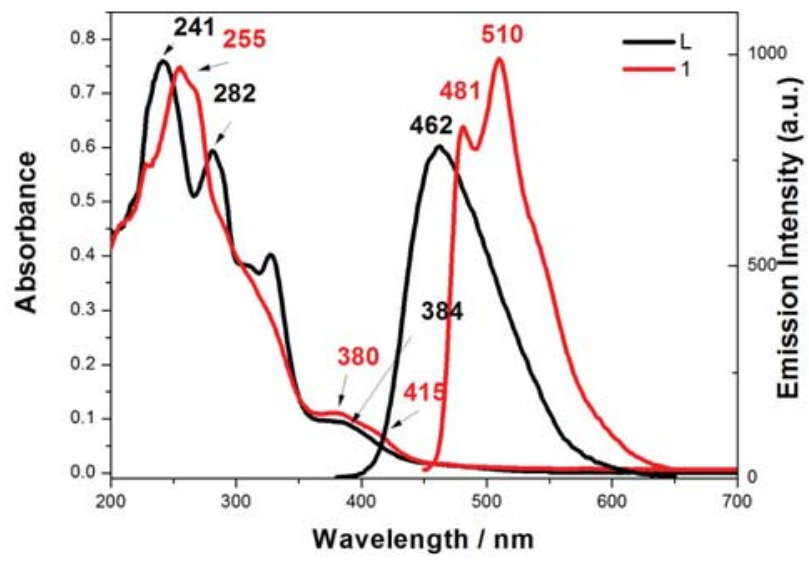

Fig. 1: UV and FL spectra of ligand $\mathbf{L}$ and complex $\mathbf{1}$ in $\mathrm{CH}_{2} \mathrm{Cl}_{2}$

try in $\mathrm{CH}_{2} \mathrm{Cl}_{2}$ solution (Fig. 2 and Table 1). Both compounds ( $\mathbf{L}$ and $\mathbf{1})$ exhibit two reversible one-electron oxidation processes, which are associated with the successive oxidation of the TTF unit to $\mathrm{TTF}^{+}$and $\mathrm{TTF}^{2+}$. Additionally, complex 1 show a irreversible oxidation peak $\left(E_{\mathrm{p}}{ }^{\text {ox }}\right)$ at $1.88 \mathrm{~V}$, which is attributed to the metal-centered $\mathrm{Ir}^{3+} / \mathrm{Ir}^{4+}$ oxidation couple. ${ }^{21,22}$ In comparison with the ligand $\mathbf{L}$, the two oxidation waves for complex $\mathbf{1}$ are shifted to more negative potentials. The observed results are different from the previous reported work, ${ }^{23}$ it is possible that the triazole-pyridine unit is grafted on the TTF core through a non-conjugated spacer group, which is disadvantageous to intramolecular electron transfer and communications. ${ }^{10}$

Table 1: Redox potentials of ligand $\mathbf{L}$ and complex $\mathbf{1}$

\begin{tabular}{lccc}
\hline Compounds & $\boldsymbol{E}_{\mathbf{1 / 2}}{ }^{\mathbf{1}}(\mathbf{V})^{\boldsymbol{a}}$ & $\boldsymbol{E}_{\mathbf{1 / 2}}{ }^{\mathbf{2}}(\mathbf{V})^{\boldsymbol{a}}$ & $\boldsymbol{E}_{p}{ }^{\mathbf{0 x}}\left(\mathbf{I r}^{\mathbf{3}+4+}\right)(\mathbf{V})$ \\
\hline $\mathbf{L}$ & 0.57 & 0.91 & - \\
$\mathbf{1}$ & 0.48 & 0.87 & 1.88 \\
\hline
\end{tabular}

${ }^{a} E_{1 / 2}=1 / 2\left(E_{\mathrm{pa}}+E_{\mathrm{pc}}\right)$, where $E_{\mathrm{pa}}$ and $E_{\mathrm{pc}}$ are the anodic and cathodic peak potentials, respectively.

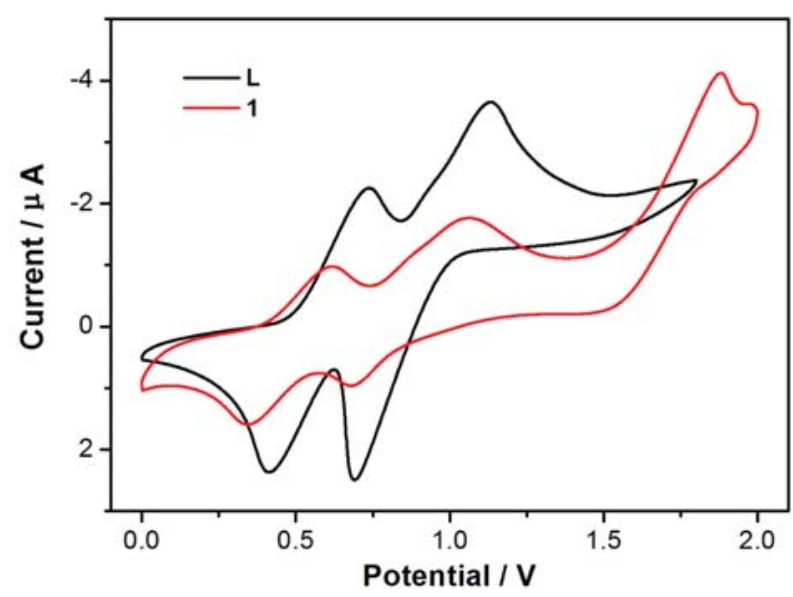

Fig. 2: Cyclic voltammograms for ligand $\mathbf{L}$ and complex $\mathbf{1}$ in $\mathrm{CH}_{2} \mathrm{Cl}_{2}$ solution containing $n-\mathrm{Bu}_{4} \mathrm{NClO}_{4}(0.1 \mathrm{M})$ at a sweep rate of $100 \mathrm{mV} / \mathrm{s}$

\section{Conclusions}

In conclusion, a new iridium(III) complex 1 based on tetrathiafulvalene-substituted triazole-pyridine ligand, has been synthesized and fully characterized by ${ }^{1} \mathrm{H}$ NMR, ${ }^{13} \mathrm{C}$ NMR, mass spectrometry, FTIR and elemental analyses. The photophysical and electrochemical properties have been measured and analyzed. The luminescent spectra show that the emissive state originates from mixed intraligand and metal-to-ligand charge transfer ${ }^{3}\left(\pi \rightarrow \pi^{*}+\right.$ MLCT) transitions. The electrochemical studies reveal that 1 undergo reversible TTF/TTF ${ }^{+} / \mathrm{TTF}^{2+}$ redox processes and one irreversible $\mathrm{Ir}^{3+} \rightarrow \mathrm{Ir}^{4+}$ oxidation process. The research plays a role in designing new photoelectric functional materials, and more work is going on in our laboratory.

\section{Acknowledgements}

This work was supported by the National Natural Science Foundation of China (No. 21501037), the Natural Science Foundation of Hainan Province (No. 20152031) and Hainan Provincial Innovation Experiment Program for University Students (No. 201511658002).

\section{Supplementary Material}

${ }^{1} \mathrm{H}$ NMR, ${ }^{13} \mathrm{C}$ NMR and ESI-MS spectra for iridium complex $\mathbf{1}$.

\section{References}

1. M. Bendikov, F. Wudl, D. F. Perepichka, Chem. Rev. 2004, 104, 4891-4945.

http://pubs.acs.org/doi/abs/10.1021/cr030666m

2. S. Wenger, P. A. Bouit, Q. L. Chen, J. Teuscher, D. D. Censo, R. H. Baker, J. E. Moser, J. L. Delgado, N. Martin, S. M. Zakeeruddin, M. Grätzel, J. Am. Chem. Soc. 2010, 132, 5164-5169.

http://pubs.acs.org/doi/abs/10.1021/ja909291h

3. F. G. Brunetti, J. L. López, C. Atienza, N. Martín, J. Mater. Chem. 2012, 22, 4188-4205.

http://pubs.rsc.org/en/content/articlepdf/2012/jm/c2jm $15710 \mathrm{a}$

4. D. Canevet, M. Sallé, G. X. Zhang, D. Q. Zhang, D. B. Zhu, Chem. Commun. 2009, 2245-2269.

5. Y. G. Sun, S. F. Ji, P. Huo, J. X. Yin, Y. D. Huang, Q. Y. Zhu, J. Dai, Inorg. Chem. 2014, 53, 3078-3087.

6. G. N. Li, L. R. He, L. Li, W. F. Cheng, X. Y. Li, H. H. Chen, Acta Chim. Slov. 2014, 61, 786-791.

https://journals.matheo.si/index.php/ACSi/article/view/473

7. S. F. Ji, Y. G. Sun, P. Huo, W. C. Shen, Y. D. Huang, Q. Y. Zhu, J. Dai, Inorg. Chem. 2014, 53, 3611-3617. 
8. J. Qin, L. Hu, N. Lei, Y. F. Liu, K. K. Zhang, J. L. Zuo, Acta Chim. Slov. 2014, 61, 740-745.

https://journals.matheo.si/index.php/ACSi/article/view/443

9. D. Lorcy, N. Bellec, M. Fourmigué, N. Avarvari, Coordin. Chem. Rev. 2009, 253, 1398-1438.

http://www.sciencedirect.com/science/article/pii/S0010854 508001768

10. G. N. Li, L. R. He, D. Xia, L. Li, W. F. Cheng, K. X. Li, F. Cui, Z. G. Niu, J. Chin. Chem. Soc. 2015, 62, 889-897. http://onlinelibrary.wiley.com/doi/10.1002/jccs.201500251 /abstract

11. K. R. J. Thomas, M. Velusamy, J. T. Lin, C. H. Chien, Y. T. Tao, Y. S. Wen, Y. H. Hu, P. T. Tai, Inorg. Chem. 2005, 44, 5677-5685.

http://pubs.acs.org/doi/abs/10.1021/ic050385s

12. M. A. Baldo, C. Adachi, S. R. Forrest, Phys. Rev. B. 2000, 62, 10967-10977.

http://journals.aps.org/prb/abstract/10.1103/PhysRevB.62. 10967\#fulltext

13. J. M. Lupton, I. D. W. Samuel, M. J. Frampton, R. Beavington, P. L. Burn, Adv. Funct. Mater. 2001, 11, 287-294. http://onlinelibrary.wiley.com/doi/10.1002/1616-3028(200 108)11:4\%3C287::AID-ADFM287\%3E3.0.CO;2-Z/abstract

14. Y. P. Zeng, C. W. Gao, L. J. Hu, H. H. Chen, G. Y. Chen, G. N. Li, Z. G. Niu, Acta Chim. Slov. 2015, 62, 917-922. https://journals.matheo.si/index.php/ACSi/article/view/1744

15. R. D. Costa, E. Ortí, H. J. Bolink, S. Graber, S. Schaffner, M. Neuburger, C. E. Housecroft, E. C. Constable, Adv. Funct. Mater. 2009, 19, 3456-3463.

http://onlinelibrary.wiley.com/doi/10.1002/adfm.200900911 /full
16. G. N. Li, Y. Liao, T. Jin, Y. Z. Li, Inorg. Chem. Commun. 2013, 35, 27-30.

http://www.sciencedirect.com/science/article/pii/S13877003 13002153

17. S. Okada, K. Okinaka, H. Iwawaki, M. Furugori, M. Hashimoto, T. Mukaide, J. Kamatani, S. Igawa, A. Tsuboyama, T. Takiguchi, K. Ueno, Dalton Trans. 2005, 9, 15-83. http://pubs.rsc.org/en/content/articlehtml/2005/dt/b417058j

18. J Qin, S. Y. Deng, C. X. Qian, T. Y. Li, H. X. Ju, J. L. Zuo, J. Organomet. Chem. 2014, 750, 7-12.

19. Z. G. Niu, D. Liu, J. Zuo, J. M. Yang, Y. H. Su, Y. D. Yang, G. N. Li, Inorg. Chem. Commun. 2014, 43, 146-150. http://www.sciencedirect.com/science/article/pii/S13877003 14000872

20. L. Y. Zhang, G. F. Liu, S. L. Zheng, B. H. Ye, X. M. Zhang, X. M. Chen, Eur. J. Inorg. Chem. 2003, 2965-2971. http://onlinelibrary.wiley.com/doi/10.1002/ejic.200300061/ abstract

21. S. Bettington, M. Tavasli, M. R.Bryce, A. Beeby, H. A. Attar, A. P. Monkman, Chem. Eur. J. 2007, 13, 1423-1431. http://onlinelibrary.wiley.com/doi/10.1002/chem.200600888 /citedby

22. M. K. Nazeeruddin, R. T. Wegh, Z. Zhou, C. Klein, Q. Wang, F. D. Angelis, S. Fantacci, M. Grätzel, Inorg. Chem. 2006, 45, 9245-9250. http://pubs.acs.org/doi/abs/10.1021/ic060495e

23. G. N. Li, T. Jin, L. Sun, J. Qin, D. Wen, J. L. Zuo, X. Z. You, J. Organomet. Chem. 2011, 696, 3076-3085. http://www.sciencedirect.com/science/article/pii/S0022328 X11003676

\section{Povzetek}

Sintetiziran in strukturno okarakteriziran je nov iridijev(III) kompleks $\left[\operatorname{Ir}(\mathrm{ppy})_{2}(\mathbf{L})\right] \mathrm{PF}_{6}(\mathbf{1}) \mathrm{z}$ vezanim triazol-piridinskim ligandom modificiranim s tetratiafulvensko skupino. Absorpcijski in luminiscenčni spekter ter elektrokemijske lastnosti $\mathbf{L}$ in $\mathbf{1}$ so bili raziskani. Kompleks $\mathbf{1}$ emitira pri sobni temperature pri 481 in $510 \mathrm{~nm}$. Široki in strukturirani emisijski trakovi so pripisani mešanju ${ }^{3} \mathrm{LC}\left({ }^{3} \pi-\pi^{*}\right)$ in ${ }^{3} \mathrm{CT}\left({ }^{3} \mathrm{MLCT}\right)$ vzbujenih stanj. Vpliv koordinacije iridijevega iona na redoks lastnosti TTF skupine je bil raziskan s pomočjo ciklične voltametrije. 